\title{
ON BASE CHANGE FOR ODD ORTHOGONAL GROUPS
}

\author{
J. W. COGDELL AND I. I. PIATETSKI-SHAPIRO
}

Let $Y$ be an orthogonal space over $\mathbb{Q}$ of rank $2 n+1 \geq 5$ and signature $(2 n, 1)$ over $\mathbb{R}$. Let $G=S O(Y)$ be the special orthogonal group of $Y$, and $G(\mathbb{A})$ its points in the adele ring $\mathbb{A}$ of $\mathbb{Q}$. Let $\pi$ be a cuspidal automorphic representation of $G(\mathbb{A})$. Then $\pi=\otimes \pi_{p}$ is a restricted tensor product of local representations with $\pi_{\infty}$ an irreducible admissible representation of $G(\mathbb{R})=$ $S O(2 n, 1)$, and for all finite $p$ not in a finite set $S$ the group $G\left(\mathbb{Q}_{p}\right)$ is split and the representation $\pi_{p}$ is unramified, i.e., there is a non-zero vector in $\pi_{p}$ fixed by the compact subgroup $G\left(\mathbb{Z}_{p}\right)$. If $K$ is a real quadratic extension of $\mathbb{Q}$ with adele ring $\mathbb{A}_{K}$, we say that an irreducible admissible representation $\Pi=\otimes \Pi_{v}$ of $G\left(\mathbb{A}_{K}\right)$ is a base change lift of $\pi$ provided that the local components $\Pi_{v}$ of $\Pi$ satisfy the following conditions:

(1) $\Pi_{\infty_{1}}$ and $\Pi_{\infty_{2}}$ have the same Langlands parameters as $\pi_{\infty}$ as representations of $S O(2 n, 1)$.

(2) For finite $p \notin S$ which split in $K, p=v_{1} v_{2}$, we have $\Pi_{v_{1}}=\Pi_{v_{2}}=\pi_{p}$.

(3) For finite $p \notin S$ which are inert in $K, \Pi_{p}$ is an unramified representation with Satake parameters given by the composition of the Satake parameters of $\pi_{p}$ with the local norm map.

The main theorem of this paper is the following:

Theorem 1. There exist a cuspidal automorphic representation $\pi$ of $G(\mathbb{A})$ and a real quadratic extension $K$ of $\mathbb{Q}$ such that no base change $\Pi$ of $\pi$ to $G\left(\mathbb{A}_{K}\right)$ is automorphic.

With the same notation, we say that an irreducible admissible representation $\Pi=\otimes \Pi_{v}$ of $G\left(\mathbb{A}_{K}\right)$ is a weak base change lift of $\pi$ provided that the local components $\Pi_{v}$ of $\Pi$ satisfy conditions (2) and (3) above, i.e., we impose local conditions at the finite unramified places only. Of course, any base change is automatically a weak base change. In the case of $2 n+1=5$ we are able to strengthen Theorem 1 by using this weaker notion of base change.

Theorem 2. Let $2 n+1=5$. There exist a cuspidal automorphic representation $\pi$ of $G(\mathbb{A})$ and a real quadratic extension $K$ of $\mathbb{Q}$ such that no weak base change $\Pi$ of $\pi$ to $G\left(\mathbb{A}_{K}\right)$ is automorphic.

Received by the editors January 24, 1994 and, in revised form, August 12, 1994.

1991 Mathematics Subject Classification. Primary 11F70, 11 F27.

The first author was supported in part by NSA grant MDA904-91-H-0040.

The second author was supported in part by NSF grant DMS- 8807336 .

(C)1995 American Mathematical Society 
One reason for the existence of these examples is that the group $G$ is not quasi-split. If we replace $G$ by the quasi-split $S O_{2 n+1}$, then an automorphic base change should exist for all quadratic extensions $K / \mathbb{Q}$. For $\mathrm{SO}_{5}$ we describe this base change in [4] (see also Section 6 of this paper).

Our results should be contrasted with the results on base change for $G L_{2}$ established by Langlands [8,14] and in general for $G L_{n}$ by Arthur and Clozel [1]. If $\pi$ is a cuspidal automorphic representation of $G L_{n}(\mathbb{A})$ and $K / \mathbb{Q}$ is any cyclic extension of prime degree, then $\pi$ always has a weak base change to $G L_{n}\left(\mathbb{A}_{K}\right)$ which is automorphic, and in fact every weak base change is automatically a strong base change in the terminology of [1], a notion of base change which imposes local conditions at all places and implies base change in our sense.

Our method of proof uses the $\Theta$-correspondence between representations of $G$ and representations of $\widetilde{S L}_{2}$, the twofold metaplectic cover of $S L_{2}$, [16], Waldspurger's results on the correspondence between representations of $\widetilde{S L}_{2}$ and representations of $P G L_{2}[15,22,23]$, and our previous results on base change for $\widetilde{S L}_{2}$ [3].

Under the same assumptions, there exist solvable extensions $K$ of $\mathbb{Q}$ of degree $2 l$, where $l$ is odd and as large as we would like, such that $\pi$ has no automorphic base change to $G\left(\mathbb{A}_{K}\right)$ as well. In our example, since the local base change always exists, we can always find an irreducible admissible representation $\Pi=\otimes \Pi_{v}$ of the adelic group $G\left(\mathbb{A}_{K}\right)$ which is a lifting of $\pi=\otimes \pi_{p}$ even if it is not automorphic. Hence it makes sense to consider a subsequent base change of $\Pi$. The image of $\Pi$ under any subsequent quadratic base change will again be automorphic. Moreover, under the natural Langlands lifting to $G L_{2 n}$ the image of $\Pi$ will also be automorphic. This is a consequence of our method of proof and the results of [3].

We would like to thank the editor and the referee for several helpful suggestions which greatly improved the exposition.

\section{BASIC DEFINITIONS}

As our definition of base change we will take the definition which is compatible with what is known about the arithmetic parameterization of admissible representations at the archimedean places (via the Langlands classification) and at the non-archimedean places which are unramified (via the Satake parameterization).

Let $K$ be a real quadratic extension of $\mathbb{Q}$. Let $\pi=\otimes \pi_{p}$ be an irreducible cuspidal automorphic representation of $G(\mathbb{A})$. Let $S$ be the finite set of places, containing $\infty$, such that for all $p \notin S$ the prime $p$ does not ramify in $K$ (i.e., is split or inert), the group $G\left(\mathbb{Q}_{p}\right)$ is split, and the representation $\pi_{p}$ is unramified. Let $\Pi=\otimes \Pi_{v}$ be an irreducible admissible representation of $G\left(\mathbb{A}_{K}\right)$.

Definition. $\Pi$ is a base change lift of $\pi$ if it satisfies the following local conditions:

(1) If $\infty_{1}$ and $\infty_{2}$ are the two archimedean places of $K$, then $\Pi_{\infty_{1}}$ and 
$\Pi_{\infty_{2}}$ are both L-equivalent to $\pi_{\infty}$, i.e., have the same Langlands parameters as $\pi_{\infty}$.

(2) For finite $p \notin S$ which split in $K, p=v_{1} v_{2}$, we have $\Pi_{v_{1}}=\Pi_{v_{2}}=\pi_{p}$.

(3) For finite $p \notin S$ which are inert in $K, \Pi_{p}$ is an unramified representation with Satake parameters given by the composition of the Satake parameters of $\pi_{p}$ with the local norm map.

$\Pi$ is a weak base change lift of $\pi$ if it satisfies the local conditions (2) and (3) at the places $p \notin S$.

Usually definitions of base change include conditions (1) through (3). The weakest possible definition would require only (2) and (3). It is clear that a base change as defined here is always a weak base change.

Let us explain the base change for the unramified finite places in a little more detail. We are interested in the case where $G_{p}=G\left(\mathbb{Q}_{p}\right)$ is quasi-split (and hence split since it is an odd dimensional orthogonal group). Let $Y$ be a quadratic space of dimension $2 n+1$ over $\mathbb{Q}$ such that $G=S O(Y)$ as an algebraic group. Since we are assuming $G_{p}$ is split at $p$, then the Witt index of $Y_{p}=Y \otimes \mathbb{Q}_{p}$ must be $n$. Decompose $\stackrel{p}{p}_{p}$ into an orthogonal direct sum

$$
Y_{p}=\mathbb{Q}_{p} e_{1} \oplus \cdots \oplus \mathbb{Q}_{p} e_{n} \oplus Y_{p}^{\prime} \oplus \mathbb{Q}_{p} e_{n}^{\prime} \oplus \cdots \oplus \mathbb{Q}_{p} e_{1}^{\prime},
$$

where $\left(e_{i}, e_{j}\right)=0=\left(e_{i}^{\prime}, e_{j}^{\prime}\right)$ and $\left(e_{i}, e_{j}^{\prime}\right)=\delta_{i, j}$, and $Y_{p}^{\prime}$ is one dimensional anisotropic. Let $B_{p}$ be the minimal parabolic subgroup associated to this decomposition. The Levi subgroup of $B_{p}$ is the maximal split torus $T_{p} \simeq$ $G L_{1}\left(\mathbb{Q}_{p}\right)^{n}$. Any spherical representation of $G_{p}$ is the unique unramified constituent of an induced representation of the form

$$
\operatorname{Ind}_{B_{p}}^{G_{p}}\left(\mu_{1} \otimes \cdots \otimes \mu_{n}\right) \text {, }
$$

where each $\mu_{i}$ is an unramified character of $G L_{1}\left(\mathbb{Q}_{p}\right) \simeq \mathbb{Q}_{p}^{\times}$[2]. The Satake class for such a representation will be a semi-simple conjugacy class in the maximal split torus of ${ }^{L} G^{\circ}=S p_{2 n}(\mathbb{C})$. For the spherical representation above it is the conjugacy class determined by

$$
t_{\pi_{p}}=\left(\begin{array}{cccccc}
\mu_{1}(p) & & & & & \\
& \ddots & & & & \\
& & \mu_{n}(p) & & & \\
& & & \mu_{n}(p)^{-1} & & \\
& & & & \ddots & \\
& & & & & \mu_{1}(p)^{-1}
\end{array}\right) .
$$

Now if $K_{v}$ is an unramified quadratic extension of $\mathbb{Q}_{p}$ and $Y_{v}=Y_{p} \otimes K_{v}$, then the base change $\Pi_{v}$ of $\pi_{p}$ will be the unique unramified constituent of the induced representation

$$
\operatorname{Ind}_{B_{v}}^{G_{v}}\left(\mu_{1} \circ N_{K_{v} / \mathbb{Q}_{p}} \otimes \cdots \otimes \mu_{n} \circ \cdot N_{K_{v} / \mathbb{Q}_{p}}\right) .
$$


Its Satake parameter will be formed from the characters $\mu_{i} \circ N_{K_{v} / \mathbb{Q}_{p}}$ evaluated at a uniformizer $\varpi_{v}$ for $K_{v}$ as above, and in fact $t_{\Pi_{v}}=t_{\pi_{p}}^{2}$.

If $p$ is an unramified place which splits into two distinct places $v_{1}$ and $v_{2}$ in $K$, so that $\mathbb{Q}_{p} \simeq K_{v_{1}} \simeq K_{v_{2}}$, then we require that $\pi_{p} \simeq \Pi_{v_{1}} \simeq \Pi_{v_{2}}$. This is consistent with the above formulation for a degree one unramified extension and in terms of Satake parameters $t_{\Pi_{v_{i}}}=t_{\pi_{p}}$ for $i=1,2$.

For our purposes it will also be convenient to have a weak notion of $\mathrm{L}$ equivalence for global representations.

Definition. Let $k$ be a number field. Let $\pi=\otimes \pi_{v}$ and $\pi^{\prime}=\otimes \pi_{v}^{\prime}$ be two irreducible automorphic representations of $G\left(\mathbb{A}_{k}\right)$. We will say that $\pi$ and $\pi^{\prime}$ are weakly $L$-equivalent if for almost all places $v$ of $k$ where $\pi$ is unramified we have $\pi_{v} \simeq \pi_{v}^{\prime}$. In particular, if $S$ is a finite set of places including the archimedean places such that $\pi_{v}$ and $\pi_{v}^{\prime}$ are unramified and $\pi_{v} \simeq \pi_{v}^{\prime}$ for those places $v \notin S$, then $L^{S}(\pi, s)=L^{S}\left(\pi^{\prime}, s\right)$ for the Langlands partial Lfunctions.

\section{SPECIAL AUTOMORPHIC FORMS AND REPRESENTATIONS}

Let $k$ be a number field. Let $Y$ denote an orthogonal space of Witt index 1 and odd dimension $2 n+1 \geq 5$ over $k$. Let $($,$) denote the non-$ degenerate symmetric bilinear pairing on $Y$ and let $q(t)=\frac{1}{2}(t, t)$ be the associated quadratic form. Since $\operatorname{Witt}(Y)=1$ we may write

$$
Y=k e_{1}+L+k e_{1}^{\prime},
$$

where $e_{1}$ and $e_{1}^{\prime}$ are isotropic satisfying $\left(e_{1}, e_{1}^{\prime}\right)=1$, so that the span $\left\langle e_{1}, e_{1}^{\prime}\right\rangle$ is a hyperbolic plane, and $L$ is an orthogonal space of dimension $2 n-1$ which is anisotropic over $k$. Let $G=S O(Y)$ and $G^{\prime}=S O(L)$ as algebraic groups over $k$.

In $G$ let $P$ be the parabolic subgroup preserving the line $\left\langle e_{1}\right\rangle$. Let $S$ be the unipotent radical of $P$. Then for each $s \in S$ there exists $a_{s} \in L$ such that the action of $S$ on $Y$ is given by

$$
s \cdot e_{1}=e_{1}, \quad s \cdot l=l+\left(a_{s}, l\right) e_{1}, \quad s \cdot e_{2}=e_{2}-a_{s}-\frac{1}{2}\left(a_{s}, a_{s}\right) e_{1} .
$$

The map $S \rightarrow L$ sending $s \mapsto a_{s}$ is an isomorphism of groups. Let the inverse of this map be denoted by $a \mapsto s_{a}$ with $a \in L$ and $s_{a} \in S$. With this notation, each $t \in L$ determines a linear character $\alpha_{t} \in \operatorname{Hom}(S, k)$ by $\alpha_{t}(s)=\left(t, a_{s}\right)$, and if $\psi$ is a non-trivial additive character of $k$, then any complex character of $S$ may be written in the form $\psi_{t}(s)=\psi\left(\alpha_{t}(s)\right)$ with $t \in L$. Let $M$ denote the Levi subgroup of $P$. Then $M$ is naturally isomorphic to $k^{\times} \times G^{\prime}$, where as before $G^{\prime}=S O(L)$. For each $l \in L$ which is not isotropic let $\mathscr{D}_{l}$ denote the stabilizer in $M$ of the character $\alpha_{l}(s)$ of $S$, or equivalently, the stabilizer in $M$ of the element $s_{l} \in S$ associated to $L$. Let $\mathscr{D}_{l}^{\circ}$ denote the connected component of the identity of $\mathscr{D}_{l}$. 
Fix a non-trivial additive character $\psi$ of $k \backslash \mathbb{A}_{k}$. If $\varphi$ is a cusp form on $G\left(\mathbb{A}_{k}\right)$, then, since $S\left(\mathbb{A}_{k}\right) \simeq L_{\mathbb{A}_{k}}$ is abelian, $\varphi$ will have a Fourier expansion

$$
\varphi(g)=\sum_{l \in L_{k}^{\prime}} \varphi_{l}(g)
$$

where $L_{k}^{\prime}=L_{k} \backslash\{0\}$ is the set of non-isotropic vectors in $L_{k}$ and

$$
\varphi_{l}(g)=\int_{S_{k} \backslash S_{\mathbf{A}_{k}}} \varphi(s g) \psi_{l}^{-1}(s) d s .
$$

It is clear that $\varphi_{l}\left(\delta_{l} g\right)=\varphi_{l}(g)$ for all $\delta_{l} \in \mathscr{D}_{l}(k)$.

Definition. A cusp form $\varphi(g)$ on $G\left(\mathbb{A}_{k}\right)$ is called special if each $\varphi_{l}(g)$ appearing in its Fourier expansion is left invariant under the adelic points of the connected stabilizer in $M$ of the associated character $\alpha_{l}$, i.e., for each $l \in L_{k}^{\prime}$ we have

$$
\varphi_{l}\left(\delta_{l} g\right)=\varphi_{l}(g) \text { for all } \delta_{l} \in \mathscr{D}_{l}^{\circ}\left(\mathbb{A}_{k}\right) \text {. }
$$

A cuspidal representation $\pi$ is called special if one (and hence all) of its cusp forms are special.

Let $\pi=\otimes \pi_{v}$ be a special cuspidal representation of $G\left(\mathbb{A}_{k}\right)$. Then each of its local components satisfies the local analog of being special, i.e., they satisfy the following uniqueness property [16].

Definition. Let $k_{v}$ be a non-archimedean local field of characteristic 0 and let $\left(\pi_{v}, V_{\pi_{v}}\right)$ be an irreducible admissible representation of $G_{v}=G\left(k_{v}\right)$. Then $\pi_{v}$ is said to have the $U$-property if for every non-isotropic $l_{v} \in L_{v}$ and every linear functional $\Lambda_{v}$ on $V_{\pi_{v}}$ satisfying $\Lambda_{v}\left(\pi_{v}\left(s_{v}\right) \xi_{v}\right)=\psi_{l_{v}}\left(s_{v}\right) \Lambda_{v}\left(\xi_{v}\right)$ for all $s_{v} \in S_{v}$ and $\xi_{v} \in V_{\pi_{v}}$ we have $\Lambda_{v}\left(\pi_{v}\left(\delta_{l_{v}}\right) \xi_{v}\right)=\Lambda_{v}\left(\xi_{v}\right)$ for all $\delta_{l_{v}} \in \mathscr{D}_{l_{v}}^{\circ}\left(k_{v}\right)$.

Proposition. A cuspidal automorphic representation $\pi^{\prime}=\otimes \pi_{v}^{\prime}$ of $G\left(\mathbb{A}_{k}\right)$ which is weakly L-equivalent to a special representation $\pi=\otimes \pi_{v}$ is again special.

Proof. Since $\pi_{v}^{\prime} \simeq \pi_{v}$ for almost all $v, \pi_{v}^{\prime}$ has the U-property for almost all $v$. Also $\pi^{\prime}$ is automorphic, so that for any Fourier coefficient of a smooth cusp form $\varphi$ in the space of $\pi^{\prime}$ we have

$$
\varphi_{l}\left(\delta_{l} g\right)=\varphi_{l}(g) \text { for all } \delta_{l} \in \mathscr{D}_{l}(k)
$$

Since $\pi_{v}^{\prime}$ has the U-property for almost all $v$, we have

$$
\varphi_{l}\left(\delta_{l, v} g_{v}\right)=\varphi_{l}\left(g_{v}\right) \text { for all } \delta_{l, v} \in \mathscr{D}_{l}^{\circ}\left(k_{v}\right)
$$

for almost all $v$. By the continuity of $\varphi_{l}$ and the approximation theorem, from (2.1) and (2.2) it follows that

$$
\varphi_{l}\left(\delta_{l} g\right)=\varphi_{l}(g) \text { for all } \delta_{l} \in \mathscr{D}_{l}^{\circ}\left(\mathbb{A}_{k}\right),
$$

and hence $\pi^{\prime}$ is special. 


\section{SPECIAL REPRESENTATIONS AND $\Theta$-CORRESPONDENCE}

Let $k$ be a number field and let $\psi$ be a non-trivial additive character of $k \backslash \mathbb{A}_{k}$. Then to this additive character there is associated both a local $\Theta$ correspondence between admissible representations of $\widetilde{S L}_{2}\left(k_{v}\right)$ and $G\left(k_{v}\right)$ and a global $\Theta$-correspondence between automorphic representations of $\widetilde{S L}\left(\mathbb{A}_{k}\right)$ and $G\left(\mathbb{A}_{k}\right)$ as in [16]. The main results of [16] can be summarized as the following.

Theorem. (i) If $v$ is a finite place of $k$, then the local $\Theta$-correspondence gives a correspondence between irreducible admissible representations of $\widetilde{S L}_{2}\left(k_{v}\right)$ and representations of $G\left(k_{v}\right)$ having the U-property. More specifically, if an irreducible admissible representation $\pi_{v}$ of $G\left(k_{v}\right)$ is a $\Theta$-lift of some irreducible admissible representation $\tau_{v}$ of $\widetilde{S L}_{2}\left(k_{v}\right)$, then $\pi_{v}$ has the U-property. Moreover, if the rank of $G_{v}$ is greater than or equal to 2 and if $\pi_{v}$ is unitary, infinite dimensional, and has the U-property, then $\pi_{v}$ is a $\Theta$-lift from $\widetilde{S L}_{2}\left(k_{v}\right)$ for some $\psi_{v}$.

(ii) The global $\Theta$-correspondence gives rise to a correspondence between cuspidal representations of $\widetilde{S L}_{2}\left(\mathbb{A}_{k}\right)$ and special representations of $G\left(\mathbb{A}_{k}\right)$. More specifically, if $\pi$ is a $\Theta$-lift of $\tau$ such that $\pi$ is a cuspidal automorphic representation of $G\left(\mathbb{A}_{k}\right)$, then $\pi$ is special. Any special automorphic representation is an image of a $\Theta$-lifting for some $\psi$.

If we combine the local $\Theta$-correspondence between $G$ and $\widetilde{S L}_{2}$ with the local $\Theta$ - or Waldspurger corrrespondence between $\widetilde{S L}_{2}$ and $P G L_{2}$, we get a characterization of unramified representations having the U-property by their Satake parameters.

Fix a non-archimedean local field $k_{v}$ of characteristic 0 . First recall that the genuine admissible representations of $\widetilde{S L}_{2}\left(k_{v}\right)$ can be parameterized by representations of $P G L_{2}\left(k_{v}\right)$. If we fix a local non-trivial additive character $\psi_{v}$, then we have a parameterization given by Waldspurger which associates to each admissible irreducible representation $\sigma_{v}$ of $P G L_{2}\left(k_{v}\right)$ a packet $W d\left(\sigma_{v}, \psi_{v}\right)$ containing one or two elements [15,23]. If $\sigma_{v}$ is unramified, then this packet is a singleton and the representation $W d\left(\sigma_{v}, \psi_{v}\right)$ is also unramified. Furthermore all genuine unramified representations of $\widetilde{S L}_{2}\left(k_{v}\right)$ occur in this way.

If $\pi_{v}$ is a unitary unramified representation of $G_{v}=G\left(k_{v}\right)$ with the Uproperty and $G_{v}$ is split, then $\pi_{v}$ is in the image of the local $\Theta$-correspondence between $\widetilde{S L}_{2}\left(k_{v}\right)$ and $G_{v}[16]$. So let us write $\pi_{v}=\Theta\left(\tau_{v}, \psi_{v}\right)$. The representation $\tau_{v}$ of $\widetilde{S L}\left(k_{v}\right)$ is necessarily unramified. If we write $\tau_{v}=W d\left(\sigma_{v}, \psi_{v}\right)$, so $\pi_{v}=\boldsymbol{\Theta}\left(\boldsymbol{W d}\left(\sigma_{v}, \psi_{v}\right), \psi_{v}\right)$, then as in [4] it is not hard to see that the correspondence $\sigma_{v} \mapsto \pi_{v}$ is independent of $\psi_{v}$. Hence the unitary, unramified representations of $G_{v}$ which have the U-property are parameterized by the unitary, unramified representations of $P G L_{2}\left(k_{v}\right)$. If $\sigma_{v}$ is an unramified representation of $P G L_{2}\left(k_{v}\right)$, let us denote $\Theta\left(W d\left(\sigma_{v}, \psi_{v}\right), \psi_{v}\right)$ by $\widetilde{\Theta}\left(\sigma_{v}\right)$. Then $\tilde{\Theta}\left(\sigma_{v}\right)$ is unramified and, in terms of Satake parameters, if the Satake parameter 
of $\sigma_{v}$ is

$$
t_{\sigma_{v}}=\left(\begin{array}{cc}
\alpha_{v} & \\
& \alpha_{v}^{-1}
\end{array}\right) \in S p_{2}(\mathbb{C}),
$$

then the Satake class of $\pi_{v}=\widetilde{\Theta}\left(\sigma_{v}\right)$ is [12]

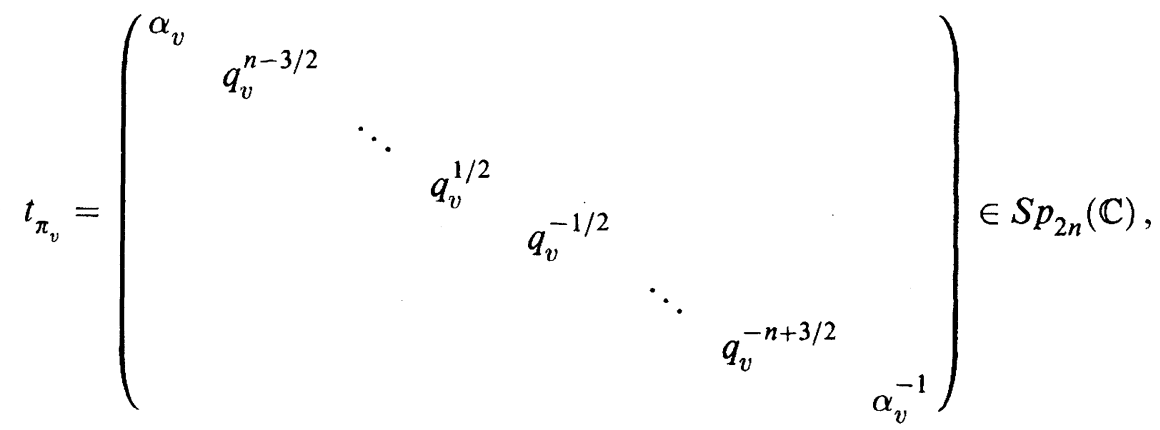

where $q_{v}$ is the order of the residue field of $k_{v}$. In terms of the local L-functions we have

$$
L\left(\pi_{v}, s\right)=L\left(\sigma_{v}, s\right) \prod_{i=0}^{n-2} \zeta_{v}(s+i+1 / 2) \zeta_{v}(s-i-1 / 2) .
$$

This discussion yields the following result.

Lemma. Let $\pi_{v}$ be an unramified representation of $G_{v}$. The Satake parameter of $\pi_{v}$ has the form (3.1) if and only if $\pi_{v}$ is a $\Theta$-lift from $\widetilde{S L}_{2}\left(k_{v}\right)$, and in this case $\pi_{v}$ has the U-property. If $\pi_{v}$ is unitary, infinite dimensional, and has the $U$-property, then its Satake parameters must be of the form (3.1).

This characterization of the unramified representations having the U-property combined with the global $\Theta$-correspondence has the following important consequence for base change.

Proposition 1. Let $\pi=\otimes \pi_{p}$ be a special automorphic representation of $G(\mathbb{A})$ and let $K$ be a real quadratic extension of $\mathbb{Q}$. Suppose that $\pi$ has a weak base change $\Pi=\otimes \Pi_{v}$ to $G\left(\mathbb{A}_{K}\right)$ which is a cuspidal automorphic representation. Then $\Pi$ is again special, i.e., in the image of the global correspondence from $\widetilde{S L}_{2}\left(\mathbb{A}_{K}\right)$.

Proof. Suppose that a weak base change $\Pi$ exists and is cuspidal. Since $\pi$ is special, then $\pi_{p}$ has the U-property for all unramified $p$. Then the Satake parameter for $\pi_{p}$ must have the form (3.1). Since the base change at the unramified places is defined by composition with the norm map, we see that the Satake parameters of $\Pi_{v}$ must also be of the form (3.1) and, in fact, $\Pi_{v}=$ $\widetilde{\Theta}\left(\left(\sigma_{p}\right)_{K_{v} / \mathbb{Q}_{p}}\right)$, where $\left(\sigma_{p}\right)_{K_{v} / \mathbb{Q}_{p}}$ denotes the local base change of $\sigma_{p}$ for $P G L_{2}$ as in $[8,14]$. Hence for almost all places, $\Pi_{v}$ has the U-property. If $\Pi$ is cuspidal, then using the approximation theorem as in Section 2 we see that $\Pi$ will be special. This implies that $\Pi$ is the $\Theta$-lift of a cuspidal automorphic representation $\Upsilon$ of $\widetilde{S L}_{2}$.

For our purposes, we will also need the following elementary global result. 
Proposition 2. Let $k=\mathbb{Q}$ and let $\tau=\otimes \tau_{p}$ be a cuspidal automorphic representation of $\widetilde{S L}_{2}(\mathbb{A})$ such that $\tau_{\infty}$ is a principal series representation induced from unitary characters. Then any non-zero $\Theta$-lift $\pi$ of $\tau$ is a cuspidal automorphic representation.

Proof. The cuspidality of the $\Theta$-lifting is implied by the compactness of the subgroup $G_{\infty}^{\prime}$, which is an anisotropic orthogonal group of dimension $2 n-1$. Since $\tau_{\infty}$ is a principal series representation and $G_{\infty}^{\prime}$ is compact, there is no local $\Theta$-lift of $\tau_{\infty}$ to $G_{\infty}^{\prime}$ [11]. Hence there can be no global $\Theta$-lift to $G^{\prime}(\mathbb{A})$ and so the $\Theta$-lift to $G(\mathbb{A})$ must be cuspidal if it is non-zero, by Rallis [17].

The case $k=\mathbb{Q}$ suffices for our purposes, even though the result is true in greater generality. It would suffice that the field $k$ have some real archimedean place $\infty$ for which $L_{\infty}$ is anisotropic and $\tau_{\infty}$ is a unitary principle series.

\section{BASE ChANGE FOR $\widetilde{S L}_{2}$}

The construction of a cuspidal representation of $G(\mathbb{A})$ which disappears under an appropriate quadratic base change is based on the similar phenomenon for $\widetilde{S L}_{2}(\mathbb{A})$ [3]. To explain this, we first need to recall some more facts about the parameterization of the representations of $\widetilde{S L}_{2}$ by those of $P G L_{2}$ given by Waldspurger [15,22,23].

Fix a local field $k_{v}$ of characteristic 0 . As we noted above, the genuine irreducible admissible representations of $\widetilde{S L}_{2}\left(k_{v}\right)$ can be parameterized by representations of $P G L_{2}\left(k_{v}\right)$. If we fix a local non-trivial additive character $\psi_{v}$, then we have a parameterization given by Waldspurger which associates to each admissible irreducible representation $\sigma_{v}$ of $P G L_{2}\left(k_{v}\right)$ a packet $W d\left(\sigma_{v}, \psi_{v}\right)$ containing one or two genuine irreducible admissible representations of $\widetilde{S L}_{2}\left(k_{v}\right)$ $[15,23]$. (Waldspurger's stated results do not quite cover all admissible representations, but they are easily extended to the other cases, as in [3].) The packets corresponding to inequivalent representations of $P G L_{2}\left(k_{v}\right)$ are disjoint, and all irreducible admissible genuine representations of $\widetilde{S L}_{2}\left(k_{v}\right)$ occur. The parameterization or partition of the admissible representations of $\widetilde{S L}_{2}\left(k_{v}\right)$ obtained in this way is compatible with the theory of L-functions for $\widetilde{S L}$ in the sense that two representations lie in the same Waldspurger packet if and only if they are L-equivalent $[3,6,7]$.

Now let $k$ be a number field and let $\psi$ be a non-trivial character of $k \backslash \mathbb{A}_{k}$. The parameterization of the irreducible genuine automorphic representations of $\widetilde{S L}_{2}\left(\mathbb{A}_{k}\right)$ by those of $P G L_{2}\left(\mathbb{A}_{k}\right)$ is more subtle. To an irreducible automorphic representation $\sigma=\otimes \sigma_{v}$ of $P G L_{2}\left(\mathbb{A}_{k}\right)$ is associated a near equivalence class, or L-packet, of irreducible automorphic representations of $\widetilde{S L}_{2}\left(\mathbb{A}_{k}\right)$ defined by

$$
\begin{aligned}
& W d(\sigma, \psi)=\left\{\tau=\otimes \tau_{v} \mid \tau\right. \text { is genuine automorphic } \\
& \text { and } \left.\tau_{v} \in W d\left(\sigma_{v}, \psi_{v}\right) \text { for all } v\right\} .
\end{aligned}
$$

All genuine automorphic representations of $\widetilde{S L}_{2}\left(\mathbb{A}_{k}\right)$ occur in some packet, but now there will be some packets which are empty since there are no genuine 
admissible representations which are both automorphic and meet all the local conditions. The representations $\sigma$ of $P G L_{2}\left(\mathbb{A}_{k}\right)$ which do not figure into the global parameterization by Waldspurger packets have the following very beautiful arithmetic characterization, due to Waldspurger $[15,23]$. The automorphic representation $\sigma$ of $P G L_{2}\left(\mathbb{A}_{k}\right)$ has an empty Waldspurger packet if and only if $\sigma$ is cuspidal and $L\left(\sigma \otimes \chi, \frac{1}{2}\right) \equiv 0$ for all quadratic characters $\chi$ of $k^{\times} \backslash \mathbb{A}_{k}^{\times}$.

Now let us turn to base change. Since the genuine automorphic representations of $\widetilde{S L}_{2}\left(\mathbb{A}_{k}\right)$ are parameterized by the automorphic representations of $P G L_{2}\left(\mathbb{A}_{k}\right)$, where base change is understood by the work of Saito-Shintani and Langlands $[8,14]$, and this parameterization is consistent with the theory of L-functions [3,6,7], the natural definition for base change for $\widetilde{S L}_{2}$ is the following [3]. If $[\tau]=W d(\sigma, \psi)$ is an L-packet for $\widetilde{S L}_{2}\left(\mathbb{A}_{k}\right)$ and $K / k$ is a cyclic extensionof prime degree, we set $[\tau]_{K / k}=W d\left(\sigma_{K / k}, \psi_{K / k}\right)$, where $\sigma_{K / k}$ is the base change of $\sigma$ as defined in $[8,14]$.

One of the main results of [3] is the following.

Theorem. The set of automorphic representations of $P G L_{2}\left(\mathbb{A}_{k}\right)$ which occur in the Waldspurger correspondence need not be preserved under quadratic base change.

This fact is responsible for several interesting phenomena [3,4]. One immediate consequence is that there can exist cuspidal automorphic representations $\tau$ of $\widetilde{S L}_{2}\left(\mathbb{A}_{k}\right)$ and quadratic extensions $K / k$ such that $[\tau]_{K / k}=\varnothing$, i.e., such that $\tau$ disappears under the quadratic base change from $k$ to $K$. We will give a more precise characterization of these $\tau$ in Section 7.

Another consequence will be Theorems 1 and 2 of this paper. They will follow from the following result.

Theorem 3. Let $k=\mathbb{Q}$ and fix a non-trivial additive character of $\mathbb{Q} \backslash \mathbb{A}$. There exist a cuspidal automorphic representation $\sigma=\otimes \sigma_{p}$ of $P G L_{2}(\mathbb{A})$ and a real quadratic extension $K$ of $\mathbb{Q}$ with the following properties:

(1) The component at infinity $\sigma_{\infty}$ is an infinite dimensional unitary principal series representation of $P G L_{2}(\mathbb{R})$.

(2) $W d(\sigma, \psi)=\{\tau\}$ is a singleton with $\tau$ cuspidal automorphic.

(3) The base change $\sigma_{K / \mathbb{Q}}$ remains cuspidal, but no longer lies in the image of the Waldspurger correspondence, i.e., $[\tau]_{K / \mathbb{Q}}=\varnothing$ and $\tau$ disappears under the base change from $\mathbb{Q}$ to $K$.

(4) Under the global $\Theta$-lift to $G(\mathbb{A}), \Theta(\tau, \psi)$ is non-zero.

In Section 7 we will give an explicit construction of a number of such representations and extensions. In the next section we will show that the representation $\pi=\boldsymbol{\Theta}(\tau, \psi)$ of $G(\mathbb{A})$ has no automorphic base change from $\mathbb{Q}$ to $K$, thereby proving Theorem 1 .

\section{Proof of TheOREM 1}

Let $\psi, K, \sigma$, and $\tau$ be as in Theorem 3 and let $\pi=\Theta(\tau, \psi)$. Since $\sigma_{\infty}$ is a unitary principle series, so is $\tau_{\infty}$ [22]. Then $\pi$ is cuspidal by Proposition 2 
of Section 3. The following two propositions show that $\pi$ has no automorphic base change to $G\left(\mathbb{A}_{K}\right)$, and hence we obtain Theorem 1 .

Proposition 1. There is no weak base change lift of $\pi$ which occurs in the space of cusp forms on $G\left(\mathbb{A}_{K}\right)$.

Proof. Suppose that a weak base change $\Pi$ exists and is cuspidal. Then Proposition 1 of Section 3 implies that $\Pi$ is the $\Theta$-lift of a cuspidal automorphic representation $\Upsilon$ of $\widetilde{S L}_{2}$. Write $\Upsilon=W d(\Sigma, \psi)$. Let $S$ be a sufficiently large finite set of places outside of which everything is unramified. Then $\Pi_{v}=\widetilde{\Theta}\left(\Sigma_{v}\right)$ for $v \notin S$, and hence its Satake parameters are given by (3.1) with

$$
\left(\begin{array}{cc}
\alpha_{v} & \\
& \alpha_{v}^{-1}
\end{array}\right)=t_{\Sigma_{v}} .
$$

On the other hand, by the definition of base change at the unramified places, the Satake parameters of $\Pi_{v}$ must be the base change of the parameters of $\pi_{p}$; that is, they must be of the form (3.1) with

$$
\left(\begin{array}{cc}
\alpha_{v} & \\
& \alpha_{v}^{-1}
\end{array}\right)=t_{\sigma_{K_{v} / Q_{p}}} .
$$

By the strong multiplicity one theorem for $P G L_{2}$ this implies that $\Sigma=\sigma_{K / \mathbb{Q}}$ is the global base change of $\sigma$ as in $[8,14]$, and $\Upsilon$ would then have to be the base change lift of $\tau$ [3]. But this contradicts the fact that $\sigma_{K / k}$ does not occur in the Waldspurger correspondence, i.e., that $\tau$ disappears under this quadratic base change.

Proposition 2. There is no base change lift of $\pi$ occurring as a subquotient of the space of automorphic forms on $G\left(\mathbb{A}_{K}\right)$.

Proof. Assume that there exists an automorphic base change lift $\Pi$ of $\pi$. By Proposition 1 it is not a cuspidal representation. Hence, by a theorem of Langlands [13], it must be a subquotient of some space of Eisenstein series associated to an induced representation of the form $\operatorname{Ind}_{P(\mathbf{A})}^{G(\mathbf{A})}\left(\rho \otimes \mu||^{s_{0}}\right)$, where $\rho$ is a cuspidal representation of $G^{\prime}(\mathbb{A}), \mu$ is a unitary character of $\mathbb{A}^{\times}$, and $s_{0} \in \mathbb{C}$.

At $\infty, \operatorname{Ind}_{P_{\infty}}^{G_{\infty}}\left(\rho_{\infty} \otimes \mu_{\infty}||^{s_{0}}\right)$ must have a constituent L-equivalent to $\Pi_{\infty}$. By definition of base change $\Pi_{\infty}$ is L-equivalent to $\pi_{\infty}$, and by construction $\pi_{\infty}$ is the local $\Theta$-lift of $\tau_{\infty}$, which is a unitary principal series for $\widetilde{S L_{2}}(\mathbb{R})$. By construction there is a unitary character $\chi_{\infty}$ such that $\tau_{\infty}=W d\left(\sigma_{\infty}, \psi_{\infty}\right)$ with $\sigma_{\infty}=\operatorname{Ind}_{B_{\infty}}^{P G L_{2}(\mathbb{R})}\left(\chi_{\infty}\right)$. Then $\Pi_{\infty}$ is L-equivalent to $\operatorname{Ind}_{P_{\infty}}^{G_{\infty}}\left(\mathbf{1}_{G_{\infty}^{\prime}} \otimes \chi_{\infty}^{\prime}\right)$, where either $\chi_{\infty}^{\prime}=\chi_{\infty}$ or $\chi_{\infty}^{\prime}=\operatorname{sgn} \cdot \chi_{\infty}$ depending on whether $2 n-1 \equiv 1(\bmod 4)$ or not [18]. Comparing infinitesimal characters, we find that $\rho_{\infty}$ and $\mathbf{1}_{G_{\infty}^{\prime}}$ agree after restriction to the connected component of $G_{\infty}^{\prime}$ and that $\chi_{\infty}^{\prime}=\mu_{\infty}||_{\infty}^{s_{0}}$. This implies that $s_{0}$ is imaginary.

Consider now a finite place where $\Pi_{v}$ is spherical. Then we know the Satake parameter of $\Pi_{v}$ has the form (3.1). Since $\pi$ was the $\Theta$-lift of a cuspidal $\tau$, then $\pi_{p}=\widetilde{\Theta}\left(\sigma_{p}\right)$ and that part of the Satake parameter for $\pi_{p}$ coming from $\sigma_{p}$ 
satisfies $\left|\alpha_{p}\right|^{ \pm}<p^{1 / 2}$ since $\sigma$ was cuspidal $[9,10]$. Then since the base change at the unramified places is given by composition with the norm map, we see that the Satake parameter for $\Pi_{v}=\widetilde{\Theta}\left(\left(\sigma_{p}\right)_{K_{v} / \mathbb{Q}_{p}}\right)$ also satisfies $\left|\alpha_{v}\right|<q_{v}^{1 / 2}$ where $\alpha_{v}$ is as in (3.1).

On the other hand, since $\Pi_{v}$ is the unramified constituent of

$$
\operatorname{Ind}_{P_{v}}^{G_{v}}\left(\rho_{v} \otimes \mu_{v}||_{v}^{s_{0}}\right) \text {, }
$$

we know that the Satake parameter for $\Pi_{v}$ must be of the form

$$
t_{\Pi_{v}}=\left(\begin{array}{lll}
\mu_{v}\left(\varpi_{v}\right)\left|\varpi_{v}\right|^{s_{0}} & & \\
& t_{\rho_{v}} & \\
& & \mu_{v}^{-1}\left(\varpi_{v}\right)\left|\varpi_{v}\right|_{v}^{-s_{0}}
\end{array}\right),
$$

where $t_{\rho_{v}}$ is the Satake parameter of $\rho_{v}$.

Comparing these two representations of the Satake parameter, we find that

$$
\mu_{v}\left(\varpi_{v}\right)\left|\varpi_{v}\right|^{s_{0}} \in\left\{\alpha_{v}^{ \pm 1}, q_{v}^{ \pm(n-3 / 2)}, \ldots, q_{v}^{ \pm 1 / 2}\right\}
$$

Since $s_{0}$ is imaginary and $\left|\mu_{v}\left(\varpi_{v}\right)\right|=1$, we must have $\mu_{v}\left(\varpi_{v}\right)\left|\varpi_{v}\right|^{s_{0}}=\alpha_{v}^{ \pm 1}$ for almost all $v$. is

Since $\Pi_{v}=\widetilde{\Theta}\left(\left(\sigma_{p}\right)_{K_{v} / k_{p}}\right)$ we see that the Satake parameter for $\Sigma_{v}=\left(\sigma_{p}\right)_{K_{v} / k_{p}}$

$$
t_{\Sigma_{v}}=\left(\begin{array}{ll}
\alpha_{v} & \\
& \alpha_{v}^{-1}
\end{array}\right)=\left(\begin{array}{ll}
\mu_{v}\left(\varpi_{v}\right) q_{v}^{s_{0}} & \\
& \mu_{v}^{-1}\left(\varpi_{v}\right) q_{v}^{-s_{0}}
\end{array}\right) .
$$

From the strong multiplicity one theorem for $P G L_{2}$, we conclude that the base change lift $\sigma_{K / k}$ of $\sigma$ is a subquotient of the space of Eisenstein series associated to $\operatorname{Ind}_{B_{2}(\mathrm{~A})}^{P G L_{2}(\mathrm{~A})}\left(\mu||^{s_{0}} \otimes \mu^{-1}||^{-s_{0}}\right)$. This contradicts our assumption, since by construction $\sigma$ remains cuspidal after base change.

\section{Proof of Theorem 2}

In this section let $2 n+1=5$. Let $\psi, K, \sigma$, and $\tau$ be as in Theorem 3 and let $\pi=\Theta(\tau, \psi)$. Since $\sigma_{\infty}$ is a unitary principle series, $\tau_{\infty}$ will also be [22]. Then $\pi$ is cuspidal by Proposition 2 of Section 3. By Proposition 1 of Section 5 we know that $\pi$ can have no weak base change to $G\left(\mathbb{A}_{K}\right)$ which is cuspidal and automorphic. Hence, to prove Theorem 2, it suffices to prove the following strengthening of Proposition 2 of Section 5.

Proposition. There is no weak base change lift of $\pi$ occurring as a subquotient of the space of automorphic forms on $G\left(\mathbb{A}_{K}\right)$.

Proof. Assume that there exists an automorphic weak base change lift $\Pi$ of $\pi$. Since it cannot be a cuspidal representation, then by the theorem of Langlands [13], it must be a subquotient of some space of Eisenstein series associated to an induced representation of the form $\operatorname{Ind}_{P(\mathbf{A})}^{G(\mathbf{A})}\left(\rho \otimes \mu||^{s_{0}}\right)$, where $\rho$ is a cuspidal representation of $G^{\prime}(\mathbb{A}), \mu$ is a unitary character of $\mathbb{A}^{\times}$, and $s_{0} \in \mathbb{C}$. 
Since $2 n+1=5, G^{\prime}$ is now a special orthogonal group in three variables over $K$. Since $K$ is a real quadratic field, the group will have signature $(3,0)$ at the archimedean places; hence $G^{\prime}$ is anisotropic over $K$. Therefore there is a division quaternion algebra $D$ over $K$ such that $G^{\prime}(K)$ is isomorphic to $P D^{\times}(K)$ [5]. At the archimedean places $D\left(K_{\infty_{i}}\right)$ must be a division algebra. At those places $v$ where $G^{\prime}\left(K_{v}\right)$ is split we have $D\left(K_{v}\right)=M_{2}\left(K_{v}\right)$ and $G^{\prime}\left(K_{v}\right) \simeq$ $P G L_{2}\left(K_{v}\right)$.

Consider now a finite place where $\Pi_{v}$ is spherical. Since $\Pi_{v}$ is the unramified constituent of $\operatorname{Ind}\left(\rho_{v} \otimes \mu_{v}||^{s_{0}}\right)$, the Satake parameter of $\Pi_{v}$ will be of the form

$$
t_{\Pi_{v}}=\left(\begin{array}{cccc}
\mu_{v}\left(\varpi_{v}\right)\left|\varpi_{v}\right|^{s_{0}} & & & \\
& \beta_{v} & & \\
& & \beta_{v}^{-1} & \\
& & & \mu_{v}^{-1}\left(\varpi_{v}\right)\left|\varpi_{v}\right|_{v}^{-s_{0}}
\end{array}\right),
$$

where the Satake parameter of $\rho_{v}$ is

$$
t_{\rho_{v}}=\left(\begin{array}{ll}
\beta_{v} & \\
& \beta_{v}^{-1}
\end{array}\right) .
$$

Since $\rho_{v}$ is an irreducible unitary unramified infinite dimensional representation of the group $G^{\prime}\left(K_{v}\right) \simeq P G L_{2}\left(K_{v}\right)$, we have the estimate $\left|\beta_{v}\right|^{ \pm 1}<q_{v}^{1 / 2}$.

On the other hand, since $\Pi$ is a weak base change of $\pi$, we know the Satake parameter of $\Pi_{v}$ has the form (3.1), i.e.,

$$
t_{\mathrm{\Pi}_{v}}=\left(\begin{array}{cccc}
\alpha_{v} & & & \\
& q_{v}^{1 / 2} & & \\
& & q_{v}^{-1 / 2} & \\
& & & \alpha_{v}^{-1}
\end{array}\right)
$$

Since $\pi$ was the $\Theta$-lift of the cuspidal $\tau$, then $\pi_{p}=\widetilde{\Theta}\left(\sigma_{p}\right)$ and $\Pi_{v}=\widetilde{\Theta}\left(\Sigma_{v}\right)$, where $\Sigma_{v}=\left(\sigma_{p}\right)_{K_{v} / \mathbb{Q}_{p}}$ and

$$
t_{\Sigma_{v}}=\left(\begin{array}{ll}
\alpha & \\
& \alpha_{v}^{-1}
\end{array}\right)
$$

Since $\sigma_{K / Q}$ is assumed to be cuspidal, we have the estimate $\left|\alpha_{v}\right|^{ \pm 1}<q_{v}^{1 / 2}$ by $[9,10]$.

If we compare the above forms of the Satake parameters for $\Pi_{v}$ for unramified $v$ we can draw the following conclusions. Since $\left|\beta_{v}\right|^{ \pm 1}<q_{v}^{1 / 2}$ we must have $\left\{\beta_{v}, \beta_{v}^{-1}\right\}=\left\{\alpha_{v}, \alpha_{v}^{-1}\right\}$, i.e., as Satake classes $t_{\rho_{v}}=t_{\Sigma_{v}}$, where $\Sigma_{v}=\left(\sigma_{p}\right)_{K_{v} / Q_{p}}$. Therefore $\rho_{v} \simeq \Sigma_{v}$ as representations of $P G L_{2}\left(K_{v}\right)$. Then we also see that we must have $s_{0}= \pm \frac{1}{2}$, and $\mu_{v}$ is trivial.

Now consider the representation $\rho$ of $P D^{\times}\left(\mathbb{A}_{K}\right)$. By the work of Jacquet and Langlands [9], we know that to $\rho$ is associated a cuspidal representation $\Sigma^{\prime}$ of $P G L_{2}\left(\mathbb{A}_{K}\right)$ such that at each place $v$ we have that $\rho_{v}=J L\left(\Sigma_{v}^{\prime}\right)$, where $J L$ 
denotes the local Jacquet-Langlands correspondence. At the unramified places $\rho_{v} \simeq \Sigma_{v}^{\prime}$ since $P D^{\times}\left(K_{v}\right) \simeq P G L_{2}\left(K_{v}\right)$.

Therefore, at almost all places we have $\Sigma_{v} \simeq \rho_{v} \simeq \Sigma_{v}^{\prime}$. By strong multiplicity one for $P G L_{2}$ we must have $\Sigma=\Sigma^{\prime}$. By construction, $\Sigma_{\infty_{i}}=\sigma_{\infty}$ is a unitary principle series. But the unitary principal series do not occur in the local Jacquet-Langlands correspondence to the local division algebra. This contradicts the fact that $\rho=J L\left(\Sigma^{\prime}\right)$.

This concludes the proof of Theorem 2 .

Consider for a moment the case of $G$ the split special orthogonal group in five variables. In this case $G \simeq P G S p_{4}$ and we have analysed the base change for the analogue of special representations, the so-called Saito-Kurokawa representations, in [4]. In this case a weak base change lift of such a $\pi$ would exist and is in fact given by a constituent of $\operatorname{Ind}\left(\rho \otimes||^{-1 / 2}\right)$ with $\rho$ equal to $\sigma_{K / \mathbb{Q}}$. (For the split $\mathrm{SO}_{5}$ the group $G^{\prime}$ occurring in the Levi supgroup of $P$ will be the split $\mathrm{SO}_{3}$ and hence isomorphic to $P G L_{2}$.)

\section{Proof of Theorem 3}

In this section we will let $k=\mathbb{Q}$ and $2 n+1 \geq 5$. Fix a non-trivial additive character $\psi$ of $\mathbb{Q} \backslash \mathbb{A}$. If $\xi \in \mathbb{Q}^{\times}$we will let $\chi_{\xi}$ denote the quadratic character of $\mathbb{Q}^{\times} \backslash \mathbb{A}^{\times}$associated to the extension $\mathbb{Q}(\sqrt{\xi})$ by class field theory.

Let us first give a more structural description of those automorphic representations of $P G L_{2}(\mathbb{A})$ which occur in the global Waldspurger correspondence with $\widetilde{S L}(\mathbb{A})$.

Let $\mathscr{A}\left(P G L_{2} / \mathbb{Q}\right)$ denote the set of irreducible automorphic representations of $P G L_{2}(\mathbb{A})$, and let $\mathscr{A}_{0}\left(P G L_{2} / \mathbb{Q}\right)$ denote the subset of irreducible cuspidal representations. Let

$$
\mathscr{A}^{\prime}\left(P G L_{2} / \mathbb{Q}\right)=\left\{\sigma \in \mathscr{A}\left(P G L_{2} / \mathbb{Q}\right): W d(\sigma, \psi) \neq \varnothing\right\}
$$

denote those automorphic representations which occur in the global Waldspurger correspondence with $\widetilde{S L}_{2}(\mathbb{A})$. Let

$$
\begin{array}{r}
\mathscr{A}_{00}\left(P G L_{2} / \mathbb{Q}\right)=\left\{\sigma \in \mathscr{A}_{0}\left(P G L_{2} / \mathbb{Q}\right) \mid \text { there exists } \xi \in \mathbb{Q}^{\times}\right. \\
\text {such that } \left.L\left(\sigma \otimes \chi_{\xi}, \frac{1}{2}\right) \neq 0\right\} .
\end{array}
$$

Then Waldspurger has shown that all irreducible non-cuspidal automorphic representations of $P G L_{2}(\mathbb{A})$ lie in $\mathscr{A}^{\prime}\left(P G L_{2} / \mathbb{Q}\right)$ and that $\mathscr{A}_{0}\left(P G L_{2} / \mathbb{Q}\right) \cap$ $\mathscr{A}^{\prime}\left(P G L_{2} / \mathbb{Q}\right)=\mathscr{A}_{00}\left(P G L_{2} / \mathbb{Q}\right)[15,23]$. This is the arithmetic characterization of those representations occurring in the Waldspurger correspondence that we mentioned in Section 4.

For our purposes, it is more convenient to use a more structural characterization of $\mathscr{A}_{00}\left(P G L_{2} / \mathbb{Q}\right)$ which is also due to Waldspurger. Given $\sigma \in$ $\mathscr{A}\left(P G L_{2} / \mathbb{Q}\right)$, let

$$
\Sigma(\sigma)=\left\{p: \sigma_{p} \text { is not a principal series representation }\right\}
$$


and let $\epsilon(\sigma)=\epsilon\left(\sigma, \frac{1}{2}\right)$, where $\epsilon(\sigma, s)$ is the $\epsilon$-factor of Jacquet and Langlands [9]. Note that for our purposes we include the one dimensional representations in the principal series representations as in [23]. Then we also have [23]

$$
\mathscr{A}_{00}\left(P G L_{2} / \mathbb{Q}\right)=\left\{\sigma \in \mathscr{A}_{0}\left(P G L_{2} / \mathbb{Q}\right): \text { either }(\mathrm{i}) \epsilon(\sigma)=1 \text { or }(\mathrm{ii}) \Sigma(\sigma) \neq \varnothing\right\} \text {. }
$$

Now let $K$ be a quadratic extension of $\mathbb{Q}$, and $\chi=\chi_{K / \mathbb{Q}}$ the quadratic character of $\mathbb{Q}^{\times} \backslash \mathbb{A}^{\times}$associated to $K$ by class field theory. Let

$$
B(K / \mathbb{Q})=\left\{\sigma \in \mathscr{A}_{00}\left(P G L_{2} / \mathbb{Q}\right): \text { (i) } \prod_{p \in \Sigma(\sigma)} \chi_{p}(-1)=-1 \text { and (ii) } \Sigma\left(\sigma_{K / \mathbb{Q}}\right)=\varnothing\right\} \text {. }
$$

Using the above structural characterization of $\mathscr{A}_{00}\left(P G L_{2} / \mathbb{Q}\right)$, one can then show that $B(K / \mathbb{Q})$ is precisely the set of $\sigma \in \mathscr{A}^{\prime}\left(P G L_{2} / \mathbb{Q}\right)$ such that $\sigma_{K / \mathbb{Q}} \notin$ $\mathscr{A}^{\prime}\left(P G L_{2} / K\right)$, i.e., the set of (necessarily cuspidal) $\sigma$ whose base change lift is not in the image of the Waldspurger correspondence. If $\sigma \in B(K / \mathbb{Q})$, then $\sigma_{K / \mathbb{Q}}$ remains a cuspidal representation of $P G L_{2}\left(\mathbb{A}_{K}\right)$. Moreover, if $L / K$ is any subsequent quadratic extension, then $\left[\sigma_{K / \mathbb{Q}}\right]_{L / K}$ is in $\mathscr{A}_{00}\left(P G L_{2} / L\right)$ and is thus again in the image of the Waldspurger correspondence. These can be found in [3].

To prove Theorem 3 , we must construct a real quadratic extension $K$ of $\mathbb{Q}$ and a representation $\sigma \in B(K / \mathbb{Q})$ which satisfies all the extra conditions listed in the statement of Theorem 3 . Our representation will be supercuspidal at one finite place and unramified at all others. We begin with the construction of the appropriate supercuspidal representation.

A local construction. Locally what we need is a supercuspidal representation $\sigma_{p}$ of $P G L_{2}\left(\mathbb{Q}_{p}\right)$ and a quadratic extension $K_{v} / \mathbb{Q}_{p}$ such that

(1) $\chi_{K_{v} / \mathbf{Q}_{p}}(-1)=-1$, and

(2) $\left(\sigma_{p}\right)_{K_{v} / \mathbb{Q}_{p}}$ is a principal series representation.

Let us first get our quadratic extension. Let $K_{v}=\mathbb{Q}_{p}(\sqrt{p})$. Let $(a, b)_{p}$ be the local Hilbert symbol of $\mathbb{Q}_{p}$. Then from [19] we know that for $a, b \in \mathbb{Q}_{p}$ we have $(a, b)_{p}=1$ iff $a$ is a norm from $\mathbb{Q}_{p}(\sqrt{b})$. Therefore $\chi_{\mathbb{Q}_{p}(\sqrt{b}) / \mathbb{Q}_{p}}(a)=$ $(a, b)_{p}$. In particular $\chi_{\mathbf{Q}_{p}(\sqrt{p}) / \mathbf{Q}_{p}}(-1)=(-1, p)_{p}$. On the other hand, the Hilbert symbol can be computed in terms of the Legendre symbol as in [19]. For $p$ an odd prime, we find $(-1, p)_{p}=(-1 / p)=(-1)^{(p-1) / 2}$. Therefore, if we take $p$ to be an odd prime, $p \equiv 3(\bmod 4)$, and our extension to be $K_{v}=\mathbb{Q}_{p}(\sqrt{p})$, then $\chi_{K_{v} / Q_{p}}(-1)=-1$. This fulfills criterion (1).

Let $\operatorname{Gal}\left(K_{v} / \mathbb{Q}_{p}\right)=\{1, l\}$. Let $\mu_{v}$ be a character of $K_{v}^{\times}$such that $\mu_{v}$ does not factor through the norm map $N_{K_{v} / \mathbb{Q}_{p}}: K_{v}^{\times} \rightarrow \mathbb{Q}_{p}^{\times}$, so $\mu_{v}^{l} \neq \mu_{v}$, and such that the restriction of $\mu_{v}$ to $\mathbb{Q}_{p}^{\times}$is the quadratic character associated to the extension $K_{v} / \mathbb{Q}_{p}$ by local class field theory, so $\mu_{v}^{l}=\mu_{v}^{-1}$. Such characters exist. Then $\mu_{v}$ determines a representation of the local Weil group $W_{\mathbb{Q}_{p}}$ by inducing $\operatorname{Ind}_{W_{\kappa_{v}}}^{W_{\mathbb{Q}_{p}}}\left(\mu_{v}\right)$. Let $\sigma_{p}\left(\mu_{v}\right)$ be the admissible representation of $P G L_{2}\left(\mathbb{Q}_{p}\right)$ 
associated to $\operatorname{Ind}_{W_{K_{v}}}^{W_{Q_{p}}}\left(\mu_{v}\right)$ by the local Langlands correspondence. This representation can be explicitly constructed using the Weil representation as in [9]. Since $\mu_{v}$ does not factor through the norm map, $\sigma_{p}\left(\mu_{v}\right)$ will be irreducible and supercuspidal. On the other hand, from either [8] or [14] we know that $\sigma_{p}\left(\mu_{v}\right)_{K_{v} / \mathbb{Q}_{p}}=\pi_{v}\left(\mu_{v}, \mu_{v}^{l}\right)$, where $\pi_{v}\left(\mu_{v}, \mu_{v}^{l}\right)$ is the principal series representation induced off the Borel as in [9]. Therefore this representation fulfills criterion (2).

There is an alternate construction of $\sigma_{p}\left(\mu_{v}\right)$ as a constituent of a representation induced from $P G L_{2}\left(\mathbb{Z}_{p}\right)$. To construct a global automorphic representation having $\sigma_{p}\left(\mu_{v}\right)$ as its local component at $p$, we will need this alternate construction. This construction can be found in Silberger [20] for example.

Let $\mathbb{Z}_{p}$ and $(p)$ denote the ring of integers and maximal ideal of $\mathbb{Q}_{p}$, and ${ }^{\circ} v$ and $\left(\varpi_{v}\right)$ the ring of integers and maximal ideal of $K_{v}$.

To $\mu_{v}$ as above, Silberger associates an irreducible unitary representation of $P G L_{2}\left(\mathbb{Z}_{p}\right)$, which we will denote $\kappa_{\mu_{v}}$. If $\mu_{v}$ has conductor $\left(\varpi_{v}\right)^{m}$, then $\kappa_{\mu_{v}}$ has conductor $(p)^{m}$ in the sense that $\kappa_{\mu_{v}}$ is trivial on the principal congruence subgroup of $P G L_{2}\left(\mathbb{Z}_{p}\right)$ of level $p^{m}$, which we denote by $K_{p}\left(p^{m}\right)$, and this is the minimal $m$ for which this is true. In our situation $m \geq 2$. Hence $\kappa_{\mu_{v}}$ factors through a representation of the finite group $P G L_{2}\left(\mathbb{Z}_{p} /(p)^{m}\right)=P G L_{2}\left(\mathbb{Z} / p^{m} \mathbb{Z}\right)$, which we will also denote $\kappa_{\mu_{v}}$.

For our problem the significance of $\kappa_{\mu_{v}}$ is the following. Let $\mu_{v}^{\prime}$ be the unique non-trivial character of $K_{v}^{\times}$such that $\mu_{v}$ and $\mu_{v}^{\prime}$ agree on o $_{v}^{\times}$and $\mathbb{Q}_{p}^{\times}$ but $\mu_{v} \neq \mu_{v}^{\prime}$, i.e., $\mu_{v}$ and $\mu_{v}^{\prime}$ differ by the unramified quadratic character of $K_{v}^{\times}$. Then

[20, Theorem (3.19)].

$$
\operatorname{Ind}_{P G L_{2}\left(\mathbb{Z}_{p}\right)}^{P G L_{2}\left(\mathbb{Q}_{p}\right)}\left(\kappa_{\mu_{v}}\right)=\sigma_{p}\left(\mu_{v}\right) \oplus \sigma_{p}\left(\mu_{v}^{\prime}\right)
$$

A global construction. Let $k=\mathbb{Q}$. Choose a prime $p_{0} \equiv 3(\bmod 4)$ and let $K=\mathbb{Q}\left(\sqrt{p_{0}}\right)$ be the associated real quadratic field. Let $v_{0}$ be the place of $K$ dividing $p_{0}$, and $K_{v_{0}}$ the completion of $K$ at $v_{0}$. Let $\mu_{v_{0}}$ be a character of $K_{v_{0}}^{\times}$as above. We would like to construct a representation $\sigma$ of $P G L_{2}(\mathbb{A})$ such that $\sigma_{\infty}$ is a unitary principal series, $\sigma_{p_{0}} \simeq \sigma\left(\mu_{v_{0}}\right)$ or $\sigma\left(\mu_{v_{0}}^{\prime}\right)$, and $\sigma_{p}$ is unramified at all $p \neq p_{0}$.

Let $\kappa_{\mu_{v_{0}}}$ be the representation of $P G L_{2}\left(\mathbb{Z}_{p}\right)$ associated to the character $\mu_{v_{0}}$ as above. Then $\kappa_{\mu_{v_{0}}}$ factors through the irreducible representation $\kappa_{\mu_{v_{0}}}$ of the finite group $P G L_{2}\left(\mathbb{Z}_{p_{0}} /\left(p_{0}\right)^{m}\right)=P G L_{2}\left(\mathbb{Z} / p_{0}^{m} \mathbb{Z}\right)$. In order to simplify notation, let $\kappa=\kappa_{\mu_{v_{0}}}$.

Let $\Gamma=P G L_{2}(\mathbb{Z})$ and let $\Gamma_{1}=\Gamma\left(p_{0}^{m}\right)$ be the kernel of the natural reduction map $P G L_{2}(\mathbb{Z}) \rightarrow P G L_{2}\left(\mathbb{Z} / p_{0}^{m} \mathbb{Z}\right)$. Via the reduction map, $\kappa$ defines a unitary representation of $\Gamma$ which is trivial on $\Gamma_{1}$. Let $V_{\kappa}$ denote the complex vector space in which $\kappa$ acts. Let $K_{\infty}=P S O(2)$, the connected component of the 


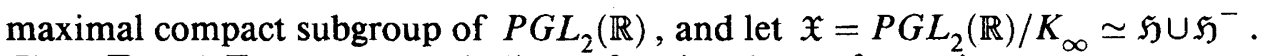
Then $\Gamma$ and $\Gamma_{1}$ act on $\mathfrak{X}$ via linear fractional transformations.

Lemma. As a representation of $\Gamma$ the finite dimensional representation $\left(\kappa, V_{\kappa}\right)$ is cuspidal in the sense that if $\sigma$ is a cusp of $\Gamma$ acting on $\mathfrak{X}$ and $\Gamma_{\sigma}$ is the stabilizer of $\sigma$ in $\Gamma$, then

$$
\sum_{\gamma \in \Gamma_{1, \sigma} \backslash \Gamma_{\sigma}} \kappa(\gamma)=0
$$

where $\Gamma_{1, \sigma}=\Gamma_{1} \cap \Gamma_{\sigma}$ is the stabilizer of $\sigma$ in $\Gamma_{1}$.

Proof. Let $B$ denote the Borel subgroup of upper triangular matrices of $P G L_{2}$ and $N$ its unipotent radical. The representation $\left(\kappa, V_{\kappa}\right)$ is cuspidal as a representation of $P G L_{2}\left(\mathbb{Z}_{p_{0}}\right)$ in the sense that it has no $N\left(\mathbb{Z}_{p_{0}}\right)$-fixed vectors [20]. Since the representation is trivial on the principal congruence subgroup $K_{p_{0}}\left(p_{0}^{m}\right)$, we see that for any $v \in V_{\kappa}$ we have

$$
\sum_{n \in\left(N\left(\mathbb{Z}_{p_{0}}\right) \cap K_{p_{0}}\left(p_{0}^{m}\right)\right) \backslash N\left(\mathbb{Z}_{p_{0}}\right)} \kappa(n) v=0
$$

since the resulting vector would be $N\left(\mathbb{Z}_{p_{0}}\right)$-fixed.

Now let $\sigma$ be a cusp of $\Gamma$. All cusps of $\Gamma$ are equivalent to the cusp at $\infty$, whose stabilizer in $P G L_{2}(\mathbb{Q})$ is $N(\mathbb{Q})$. So $\Gamma_{\infty}=N(\mathbb{Z})$. Let $\gamma_{\sigma} \in \Gamma$ be such that $\gamma_{\sigma}^{-1} \sigma=\infty$. Then the stabilizer of $\sigma$ is $\gamma_{\sigma} N(\mathbb{Q}) \gamma_{\sigma}^{-1}$ and the stabilizer in $\Gamma$ is $\Gamma_{\sigma}=\gamma_{\sigma} \Gamma_{\infty} \gamma_{\sigma}^{-1}$. Since $\Gamma_{1}$ is normal in $\Gamma, \Gamma_{1, \sigma}=\gamma_{\sigma} \Gamma_{1, \infty} \gamma_{\sigma}^{-1}$.

Let $v \in V_{\kappa}$. Then we have

$$
\sum_{\gamma \in \Gamma_{1, \sigma} \backslash \Gamma_{\sigma}} \kappa(\gamma) v=\kappa\left(\gamma_{\sigma}\right) \sum_{\Gamma_{1, \infty} \backslash \Gamma_{\infty}} \kappa(\gamma) \kappa\left(\gamma_{\sigma}^{-1}\right) v .
$$

If we let $v_{\sigma}=\kappa\left(\gamma_{\sigma}^{-1}\right) v$ and note that

$$
\Gamma_{1, \infty} \backslash \Gamma_{\infty} \simeq N\left(\mathbb{Z} / p_{0}^{m} \mathbb{Z}\right) \simeq\left(N\left(\mathbb{Z}_{p_{0}}\right) \cap K_{p_{0}}\left(p_{0}^{m}\right)\right) \backslash N\left(\mathbb{Z}_{p_{0}}\right)
$$

we see that

$$
\sum_{\Gamma_{1, \infty} \backslash \Gamma_{\infty}} \kappa(\gamma) v_{\sigma}=\sum_{\left(N\left(\mathbb{Z}_{p_{0}}\right) \cap K_{p_{0}}\left(p_{0}^{m}\right)\right) \backslash N\left(\mathbb{Z}_{p_{0}}\right)} \kappa(n) v_{\sigma}
$$

But as we noted above, this last sum must be zero. Hence as an element of $\operatorname{End}\left(V_{\kappa}\right)$ we have

$$
\sum_{\Gamma_{1, \sigma} \backslash \Gamma_{\sigma}} \kappa(\gamma)=0
$$

and $\left(\kappa, V_{\kappa}\right)$ is cuspidal as a representation of $\Gamma$.

Consider the space of $L^{2}$ vector valued functions $L^{2}\left(\Gamma_{1} \backslash \mathfrak{X}, V_{\kappa}\right)$ from $\Gamma_{1} \backslash \mathfrak{X}$ to $V_{\kappa}$. There is a natural action of $\Gamma$ on this space by left translation

$$
L(\gamma) F(z)=F\left(\gamma^{-1} x\right)
$$


for $\gamma \in \Gamma$ and $x \in \mathfrak{X}$. Since $\Gamma_{1}$ is normal in $\Gamma$, this action preserves $L^{2}\left(\Gamma_{1} \backslash \mathfrak{X}, V_{\kappa}\right)$ and factors to an action of $\Gamma / \Gamma_{1} \simeq P G L_{2}\left(\mathbb{Z} / p_{0}^{m} \mathbb{Z}\right)$. Let $P_{\kappa}$ denote the projection to the $\kappa$-isotypic component

$$
P_{\kappa} F(x)=\sum_{\gamma \in \Gamma / \Gamma_{1}} \kappa(\gamma) F\left(\gamma^{-1} x\right)
$$

The image of $P_{\kappa}$ consists of those $F \in L^{2}\left(\Gamma_{1} \backslash \mathfrak{X}, V_{\kappa}\right)$ which satisfy $F(\gamma x)=$ $\kappa(\gamma) F(x)$ for all $\gamma \in \Gamma$. Let us denote this image by $L^{2}\left(\Gamma_{1} \backslash \mathfrak{X}, V_{\kappa}\right)_{\kappa}$, or $L_{\kappa}^{2}$ for short.

Fix an orthonormal basis of $V_{\kappa}$. Then each function $F \in L_{\kappa}^{2}$ determines a vector $\left(f_{i}(x)\right)$ of component functions with each $f_{i}(x)$ in $L^{2}\left(\Gamma_{1} \backslash \mathfrak{X}\right)$. Let $A_{0}(\kappa)$ be the subspace of $L^{2}\left(\Gamma_{1} \backslash \mathfrak{X}\right)$ spanned by the coeffecient functions of all $F(x)$ in $L_{\kappa}^{2}$.

Lemma. $A_{0}(\kappa)$ is contained in the space of cuspidal automorphic functions $L_{0}^{2}\left(\Gamma_{1} \backslash \mathfrak{X}\right)$.

Proof. Let $\sigma$ be a cusp for $\Gamma_{1}$ and let $N_{\sigma}$ be the unipotent subgroup of $P G L_{2}(\mathbb{R})$ fixing $\sigma$. Then we must show that

$$
\int_{\Gamma_{1, \sigma} \backslash N_{\sigma}} f(n x) d n \equiv 0
$$

for $f \in A_{0}(\kappa)$. It is enough to consider $f(x)$ a coeffecient of $F(x) \in L_{\kappa}^{2}$. For $F(x)$ we have

$$
\begin{aligned}
\int_{\Gamma_{1, \sigma} \backslash N_{\sigma}} F(n x) & =\int_{\Gamma_{\sigma} \backslash N_{\sigma}}\left(\sum_{\Gamma_{1, \sigma} \backslash \Gamma_{\sigma}} F(\gamma n x)\right) d n \\
& =\int_{\Gamma_{\sigma} \backslash N_{\sigma}}\left(\sum_{\Gamma_{1, \sigma} \backslash \Gamma_{\sigma}} \kappa(\gamma) F(n x)\right) d n .
\end{aligned}
$$

But for each $n$ and $x, v=F(n x) \in V_{\kappa}$ and so the inner summation $\sum \kappa(\gamma) F(n x)=0$ since $\kappa$ is cuspidal as a representation of $\Gamma$. Hence

$$
\int_{\Gamma_{1, \sigma} \backslash N_{\sigma}} F(n x) d n=0
$$

and consequently

$$
\int_{\Gamma_{1, \sigma} \backslash N_{\sigma}} f(n x) d n=0 .
$$

Thus $A_{0}(\kappa) \subset L_{0}^{2}\left(\Gamma_{1} \backslash \mathfrak{X}\right)$.

Lemma. The space $A_{0}(\kappa)$ is infinite dimensional.

Proof. It suffices to show that $L_{\kappa}^{2}$ is infinite dimensional. 
Let $H$ denote the finite group $\Gamma_{1} \backslash \Gamma \simeq P G L_{2}\left(\mathbb{Z} / p_{0}^{m} \mathbb{Z}\right)$. Then $H$ acts in a natural way on $\Gamma_{1} \backslash \mathfrak{X}$, namely, if $x \in \mathfrak{X}$ and $\gamma \in \Gamma$, then the natural action $x \mapsto \gamma x$ of $\Gamma$ on $\mathfrak{X}$ factors to an action of $\Gamma_{1} \backslash \Gamma=H$ on $\Gamma_{1} \backslash \mathfrak{X}$ since $\Gamma_{1}$ is normal in $\Gamma$.

Let $x \in \mathfrak{X}$ be a point whose orbit $H x$ under $H$ has exactly $|H|$ distinct points. Then we can identify the orbit $H x$ with $H$ in the natural way. If we restrict the functions $F \in L^{2}\left(\Gamma_{1} \backslash \mathfrak{X}, V_{\kappa}\right)$ to the set $H x \cong H$, we obtain all functions $f: H \rightarrow V_{\kappa}$, which we denote by $\mathscr{F}(H, V \kappa)$. This restriction map, which we denote by $r_{x}$, intertwines the action of $H$ on $L^{2}\left(\Gamma_{1} \mathfrak{X}, V_{\kappa}\right)$ via left translation $L$ with the left regular representation of $H$ on $\mathscr{F}\left(H, V_{\kappa}\right)$. As a representation of the finite group $H, \mathscr{F}\left(H, V_{\kappa}\right)$ is isomorphic to $d_{\kappa}=\operatorname{dim} V_{\kappa}$ copies of the regular representation of $H$ on $\mathbb{C}[H]$. So we have

$$
L^{2}\left(\Gamma_{1} \backslash \mathfrak{X}, V_{\kappa}\right) \stackrel{r_{x}}{\longrightarrow} d_{\kappa} \mathbb{C}[H] .
$$

Under the map $r_{x}$, the projection $P_{\kappa}$ induces on $\mathbb{C}[H]$ the projection onto the $\kappa$-isotypic component of the regular representation of $H$. The regular representation contains $\kappa$ with multiplicity $d_{\kappa}$. Hence

$$
L^{2}\left(\Gamma_{1} \backslash \mathfrak{X}, V_{\kappa}\right)_{\kappa} \stackrel{r_{x}}{\longrightarrow} d_{\kappa}^{2} \kappa,
$$

so that $\operatorname{dim}\left(L^{2}\left(\Gamma_{1} \backslash \mathfrak{X}, V_{\kappa}\right)_{\kappa}\right) \geq d_{\kappa}^{3}$.

If we repeat this with $N$ distinct points $x_{1}, \ldots, x_{N}$ which are inequivalent mod $\Gamma$ and each of whose orbits under $H$ contains exactly $|H|$ points, then the maps $r_{x_{i}}$ are independent and we obtain $\operatorname{dim}\left(L^{2}\left(\Gamma_{1} \backslash \mathfrak{X}, V_{\kappa}\right)_{\kappa}\right) \geq N d_{\kappa}^{3}$. Hence $L^{2}\left(\Gamma_{1} \backslash \mathfrak{X}, V_{\kappa}\right)_{\kappa}$ is infinite dimensional, and so is $A_{0}(\kappa)$.

In $P G L_{2}\left(\mathbb{A}_{f}\right)$, where $\mathbb{A}_{f}$ denotes the finite adeles, consider the following open compact subgroup. Let $K_{f}=\prod K_{p}$, where $K_{p}=P G L_{2}\left(\mathbb{Z}_{p}\right)$ if $p \neq p_{0}$ and $K_{p_{0}}=K_{p_{0}}\left(p_{0}^{m}\right)$. Then $\Gamma\left(p_{0}^{m}\right)=P G L_{2}(\mathbb{Q}) \cap P G L_{2}(\mathbb{R}) K_{f}$.

Lemma. $\Gamma\left(p_{0}^{m}\right) \backslash P G L_{2}(\mathbb{R}) \cong P G L_{2}(\mathbb{Q}) \backslash P G L_{2}(\mathbb{A}) / K_{f}$.

Proof. Let $K_{f}^{\prime}=\prod P G L_{2}\left(\mathbb{Z}_{p}\right)$ be the product of the maximal compact subgroups at all finite places. By strong approximation for $S L_{2}$ and the fact the field $\mathbb{Q}$ has class number one, we know that

$$
P G L_{2}(\mathbb{Z}) \backslash P G L_{2}(\mathbb{R}) \cong P G L_{2}(\mathbb{Q}) \backslash P G L_{2}(\mathbb{A}) / K_{f}^{\prime} .
$$

Since $K_{f}$ is a normal subgroup of $K_{f}^{\prime}$, even though strong approximation fails for $P G L_{2}$, we still have

$$
P G L_{2}(\mathbb{Q}) \backslash P G L_{2}(\mathbb{A}) / K_{f} \cong \coprod_{i=1}^{c} \Gamma\left(p_{0}^{m}\right) \backslash P G L_{2}(\mathbb{R})
$$

for some integer $c$. We wish to determine $c$.

Let us first compute the number of connected components of the set

$$
P G L_{2}(\mathbb{Q}) \backslash P G L_{2}(\mathbb{A}) / K_{f} .
$$


This will be given by $\left|P G L_{2}(\mathbb{Q}) \backslash P G L_{2}(\mathbb{A}) / P G L_{2}^{+}(\mathbb{R}) K_{f}\right|$ where $P G L_{2}^{+}(\mathbb{R})$ is the connected component of $P G L_{2}(\mathbb{R})$ consisting of those elements represented by matrices with positive determinant. Since $S L_{2}$ has strong approximation, we can compute this order from its image under the determinant map. $\operatorname{det}\left(P G L_{2}(\mathbb{A})\right)=\mathbb{A}^{\times} /\left(\mathbb{A}^{\times}\right)^{2}$ consists of all square classes in $\mathbb{A}^{\times}$while $\operatorname{det}\left(P G L_{2}(\mathbb{Q}) P G L_{2}^{+}(\mathbb{R}) K_{f}\right)$ consists of those square classes determined by the subgroup $\mathbb{Q}^{\times} \mathbb{R}^{+} U_{f} \subset \mathbb{A}^{\times}$, where $U_{f}=\left(\prod_{p \neq p_{0}} \mathbb{Z}_{p}^{\times}\right) U_{p_{0}}(m)$ and $U_{p_{0}}(m)=$ $1+p_{0}^{m} \mathbb{Z}_{p_{0}}$. Since $\mathbb{Q}$ has class number one, $\mathbb{A}^{\times}=\mathbb{Q}^{\times} \mathbb{R}^{+} \Pi \mathbb{Z}_{p}^{\times}$. Since for $m \geq 1$ we have $U_{p_{0}}(m) \subset\left(\mathbb{Z}_{p_{0}}^{\times}\right)^{2}$, we see that

$$
\left|P G L_{2}(\mathbb{Q}) \backslash P G L_{2}(\mathbb{A}) / P G L_{2}^{+}(\mathbb{R}) K_{f}\right|=\left|\mathbb{Z}_{p_{0}}^{\times} /\left(\mathbb{Z}_{p_{0}}^{\times}\right)^{2}\right|=2 .
$$

Now, since $p_{0} \equiv 3(\bmod 4)$, we know that -1 is not a square $\bmod p_{0}$ (and in fact $\left.\mathbb{Z}_{p_{0}}^{\times} /\left(\mathbb{Z}_{p_{0}}^{\times}\right)^{2} \cong\{ \pm 1\}\right)$. One consequence of this is that $\Gamma\left(p_{0}^{m}\right)$ cannot contain elements represented by matrices with negative determinants. Hence $\Gamma\left(p_{0}^{m}\right) \subset P S L_{2}(\mathbb{Z})$. Therefore if we consider the quotient $\Gamma\left(p_{0}^{m}\right) \backslash P G L_{2}(\mathbb{R})$ we see that this has two connected components, distinguished by the sign of the determinant.

Therefore, comparing the number of connected components, when $p_{0} \equiv 3$ $(\bmod 4)$ we have $c=1$. This proves the Lemma. (Note that if $p_{0} \equiv 1$ $(\bmod 4)$, then $\Gamma\left(p_{0}^{m}\right)$ would contain elements whose determinants had both positive and negative signs. In this case $\Gamma\left(p_{0}^{m}\right) \backslash P G L_{2}(\mathbb{R})$ would be connected and we would have $c=2$.)

By this Lemma, we may view $A_{0}(\kappa)$ as a subspace of $L_{0}^{2}\left(P G L_{2}(\mathbb{Q}) \backslash P G L_{2}(\mathbb{A})\right)$ consisting of functions which are invariant under the action of $K=K_{\infty} K_{f}$.

By the spectral theorem for $L^{2}\left(\Gamma\left(p_{0}^{m}\right) \backslash \mathfrak{X}\right)$ this space first decomposes into a direct sum of finite dimensional eigenspaces for the Laplacian with eigenvalues occurring discretely in $[0, \infty)$ [21]. Each eigenspace for the Laplacian will be preserved by the Hecke operators $T_{p}$ for $p \neq p_{0}$ and give a finite dimensional representation of the Hecke algebra at $p_{0}$ of level $p_{0}^{m}$. We can then find a basis for $L_{0}^{2}\left(\Gamma\left(p_{0}^{m}\right) \backslash \mathfrak{X}\right)$ consisting of simultaneous eigenfunctions for the Laplacian and the Hecke operators $T_{p}$ for $p \neq p_{0}$, and generating irreducible representations of the Hecke algebra at $p_{0}$ of level $p_{0}^{m}$. Let $\left\{\varphi_{j}\right\}$ denote this basis.

If $\varphi \in A_{0}(\kappa)$, then $\varphi$ will have a spectral expansion $\varphi=\sum c_{j} \varphi_{j}$. By construction, any function $\varphi \in A_{0}(\kappa)$ under the action of the Hecke algebra at $p_{0}$ of level $p_{0}^{m}$ will generate a finite dimensional representation which must be equivalent to a sum of constituents of the representation of the Hecke algebra in the space of $K_{p_{0}}$-fixed vectors in $\operatorname{Ind}_{P G L_{2}\left(\mathbb{Z}_{p_{0}}\right)}^{P G L_{2}\left(\mathbb{P}_{p}\right)}(\kappa)$, that is, the representation of the Hecke algebra of level $p_{0}^{m}$ on $\sigma\left(\mu_{v_{0}}\right)^{K_{p_{0}}}$ or $\sigma\left(\mu_{v_{0}}^{\prime}\right)^{K_{p_{0}}}$. Hence in the spectral expansion, $c_{j}=0$ unless $\varphi_{j}$ generates a representation of the Hecke algebra of level $p_{0}^{m}$ corresponding to $\sigma\left(\mu_{v_{0}}\right)^{K_{p_{0}}}$ or $\sigma\left(\mu_{v_{0}}^{\prime}\right)^{K_{p_{0}}}$. 
Since $A_{0}(\kappa)$ is infinite dimensional, we can choose a $\varphi \in A_{0}(\kappa)$ so that not all $\varphi$ occurring in its spectral expansion have Laplace eigenvalues corresponding to complementary series representations of $P G L_{2}(\mathbb{R})$. If a $\varphi_{j}$ does not correspond to a complementary series representation, then it must correspond to a unitary principal series representation.

Therefore we can find a non-zero $\varphi_{j}$ occurring in the spectral expansion of some $\varphi \in A_{0}(\kappa)$ such that (i) the eigenvalue of the Laplacian for $\varphi_{j}$ corresponds to a unitary principal series representation, (ii) $\varphi_{j}$ is an eigenvector for the Hecke operators $T_{p}$ for $p \neq p_{0}$, and (iii) under the Hecke algebra at $p_{0}$ of level $p_{0}^{m}$ the function $\varphi_{j}$ generates a representation isomorphic to that on $\sigma\left(\mu_{v_{0}}\right)^{K_{p_{0}}}$ or $\sigma\left(\mu_{v_{0}}^{\prime}\right)^{K_{p_{0}}}$. Let $\sigma$ be the subrepresentation of $L_{0}^{2}\left(P G L_{2}(\mathbb{Q}) \backslash P G L_{2}(\mathbb{A})\right)$ generated by such a $\varphi_{j}$. Then (i)-(iii) imply that this representation is irreducible and that if we write $\sigma=\otimes \sigma_{p}$, then (i) $\sigma_{\infty}$ is the unitary principal series attached to the eigenvalue of the Laplacian on $\varphi_{j}$, (ii) for $p \neq p_{0}$ the representation $\sigma_{p}$ is the unramified representation attached to the Hecke eigenvalue of $\varphi_{j}$, and (iii) $\sigma_{p_{0}}$ is either $\sigma\left(\mu_{v_{0}}\right)$ or $\sigma\left(\mu_{v_{0}}^{\prime}\right)$. Then $\sigma$ is cuspidal, and in fact $\sigma \in A_{00}\left(P G L_{2} / \mathbb{Q}\right)$ since $\Sigma(\sigma)=\left\{p_{0}\right\} \neq \varnothing$.

Thus we see that for the $\sigma$ constructed here and $K=\mathbb{Q}\left(\sqrt{p_{0}}\right)$ we have $\Sigma\left(\sigma_{K / \mathbb{Q}}\right)=\varnothing$ and $\chi_{K_{v_{0}} / \mathbb{Q}_{p_{0}}}(-1)=-1$. Therefore $\sigma \in B(K / \mathbb{Q})$. Moreover, $\sigma_{\infty}$ is a unitary principal series representation.

Proof of Theorem 3. To obtain Theorem 3, we first take $\sigma$ as constructed above and let $\tau=W d(\sigma, \psi)$. Since $|\Sigma(\sigma)|=1, W d(\sigma, \psi)$ is a singleton [15,23]. If $2 n+1 \geq 7$, then by a result of Rallis [17] the $\theta$-lift $\Theta\left(\tau, \psi^{\prime}\right)$ is non-zero for any choice of $\psi^{\prime}$. In particular, $\pi=\Theta(\tau, \psi) \neq 0$. The Satake parameters of $\sigma_{v}$ and $\pi_{v}$ are then related as in Section 3. This proves Theorem 3 in the case $2 n+1 \geq 7$.

In the case $2 n+1=5$ there is an extra complication. There is now an additional condition for $\pi=\Theta(\tau, \psi)$ not to vanish. We need that some automorphic form $\varphi \in \tau$ has a non-vanishing $\psi_{a}$-Fourier coefficient for some $a \in q(L)$ [16], where $\psi_{a}$ is the additive character given by $\psi_{a}(x)=\psi(a x)$. Choose some $a \in \mathbb{Q}^{\times}$such that $\varphi$ has a non-vanishing $\psi_{a}$-Fourier coefficient. Write $a=a_{1} \cdot a_{2}$ with $a_{2} \in q(L)$. Then $\psi_{a}=\left(\psi_{a_{1}}\right)_{a_{2}}$ and so $\Theta\left(\tau, \psi_{a_{1}}\right) \neq 0$. Let

$$
\pi^{\prime}=\Theta\left(\tau, \psi_{a_{1}}\right)=\Theta\left(W d(\sigma, \psi), \psi_{a_{1}}\right)
$$

Now, analyzing the variation of the lifts under a change in additive character as in [23] and [4], we have

$$
\begin{aligned}
\pi^{\prime} & =\boldsymbol{\Theta}\left(W d(\sigma, \psi), \psi_{a_{1}}\right)=\boldsymbol{\Theta}\left(W d(\sigma, \psi)^{a_{1}}, \psi\right) \\
& =\Theta\left(W d\left(\sigma, \psi_{a_{1}}\right), \psi\right)=\Theta\left(W d\left(\sigma \otimes \chi_{a_{1}}, \psi\right), \psi\right),
\end{aligned}
$$

where $\chi_{\xi}$ for $\xi \in \mathbb{Q}$ denotes the quadratic character of $\mathbb{Q}^{\times} \backslash \mathbb{A}^{\times}$associated to the quadratic extension $\mathbb{Q}(\sqrt{\xi})$ of $\mathbb{Q}$ by class field theory. Now set $\sigma^{\prime}=$ 
$\sigma \otimes \chi_{a_{1}}$ and $\tau^{\prime}=W d(\sigma, \psi)$, so $\pi^{\prime}=\Theta\left(\tau^{\prime}, \psi\right)$. We claim that the statement of Theorem 3 is true for this choice of $\tau^{\prime}, \sigma^{\prime}$, and $\pi^{\prime}$.

By construction, we have

$$
\begin{aligned}
\sigma & \in B(K / \mathbb{Q}) \\
& =\left\{\rho \in \mathscr{A}_{00}\left(P G L_{2} / \mathbb{Q}\right): \text { (i) } \prod_{p \in \Sigma(\rho)} \chi_{p}(-1)=-1 \text { and (ii) } \Sigma\left(\rho_{K / \mathbb{Q}}\right)=\varnothing\right\} .
\end{aligned}
$$

The arithmetic characterization of the space $\mathscr{A}_{00}\left(P G L_{2} / \mathbb{Q}\right)$, namely

$$
\begin{aligned}
\mathscr{A}_{00}\left(P G L_{2} / \mathbb{Q}\right)=\left\{\rho \in \mathscr{A}_{0}\left(P G L_{2} / \mathbb{Q}\right) \mid \text { there exists } \xi\right. \text { such that } \\
\left.L\left(\rho \otimes \chi_{\xi}, 1 / 2\right) \neq 0\right\},
\end{aligned}
$$

is stable under quadratic twists, so that $\sigma^{\prime} \in \mathscr{A}_{00}\left(P G L_{2} / \mathbb{Q}\right)$. Since $\sigma^{\prime}=\sigma \otimes \chi_{a_{1}}$ we have $\Sigma\left(\sigma^{\prime}\right)=\Sigma(\sigma)$, and since $\left(\sigma^{\prime}\right)_{K / \mathbb{Q}}=\sigma_{K / \mathbb{Q}} \otimes\left(\chi_{a_{1}} \circ N_{K / \mathbb{Q}}\right)$ we see that $\Sigma\left(\sigma_{K / \mathbb{Q}}^{\prime}\right)=\Sigma\left(\sigma_{K / \mathbb{Q}}\right)$. Thus $\sigma^{\prime} \in B(K / \mathbb{Q})$. This shows that $\sigma^{\prime}$, $\tau^{\prime}$, and $\pi^{\prime}$ satisfy (2), (3), and (4).

On the other hand, since $\sigma_{\infty}$ is a unitary principle series, so is $\sigma_{\infty}^{\prime}=\sigma_{\infty} \otimes$ $\chi_{a_{1}, \infty}$, and $\sigma^{\prime}$ satisfies (1). Thus this choice of $\sigma^{\prime}, \tau^{\prime}$, and $\pi^{\prime}$ now satisfy the requirements of Theorem 3 in the case $2 n+1=5$.

\section{REFERENCES}

1. J. Arthur and L. Clozel, Simple algebras, base change, and the advanced theory of the trace formula, Ann. of Math. Stud., no. 120, Princeton Univ. Press, Princeton, NJ, 1989.

2. P. Cartier, Representations of p-adic groups: a survey, Proc. Sympos. Pure Math., vol. 33, part 1, Amer. Math. Soc., Providence, RI, 1979, pp. 111-155.

3. J. Cogdell and I.I. Piatetski-Shapiro, Base change for $\widetilde{S L}_{2}$, J. Number Theory 27 (1987), 285-303.

4. Base change for the Saito-Kurokawa representations of $P G S p(4), \mathrm{J}$. Number Theory 30 (1988), 298-320.

5. M. Eichler, Quadratische Formen und orthogonale Gruppen, Springer-Verlag, Berlin, 1952.

6. S. Gelbart and I.I. Piatetski-Shapiro, On Shimura's correspondence for modular forms of half-integral weight, Automorphic Forms, Representation Theory, and Arithmetic (Procedings of the Bombay Colloquium, 1979), Springer-Verlag, Berlin, 1981, pp. 1-39.

7. Shimura, Israel J. Math. 44 (1983), 97-125.

8. P. Gérardin and J.P. Labesse, The solution of a base change problem for GL(2), Proc. Sympos. Pure Math., vol. 33, part 2, Amer. Math. Soc., Providence, RI, 1979, pp. 115-133.

9. H. Jacquet and R.P. Langlands, Automorphic forms on $G L(2)$, Lecture Notes in Math., vol. 114, Springer-Verlag, Berlin, 1970.

10. H. Jacquet and J. Shalika, On Euler products and the classification of automorphic representations. I, Amer. J. Math. 103 (1981), 449-558.

11. M. Kashiwara and M. Vergne, On the Segal-Shale-Weil representation and harmonic polynomials, Invent. Math. 44 (1978), 1-47.

12. S.S. Kudla, On the local theta correspondence, Invent. Math. 83 (1986), 229-255.

13. R.P. Langlands, On the notion of an automorphic representation, Proc. Sympos. Pure Math., vol. 33, part 1, Amer. Math. Soc., Providence, RI, 1979, pp. 203-207. 
14. _ Base change for $G L(2)$, Ann. of Math. Stud., no. 96, Princeton Univ. Press, Princeton, NJ, 1980.

15. I.I. Piatetski-Shapiro, Work of Waldspurger, Lie Group Representations. II (Proceedings, University of Maryland 1982-83), Lecture Notes in Math., vol. 1041, Springer-Verlag, Berlin, 1984, pp. 280-302.

16. 1.I. Piatetski-Shapiro and D. Soudry, Special representations of rank one orthogonal groups, Israel J. Math. 64 (1988), 276-314.

17. S. Rallis, Injectivity properties of liftings associated to Weil representations, Compositio Math. 52 (1984), 139-169.

18. S. Rallis and G. Schiffmann, Weil representation. I: Intertwining distributions and discrete spectrum, Mem. Amer. Math. Soc. No. 231 (1980).

19. J.-P. Serre, A course in arithmetic, Graduate Texts in Math., no. 7, Springer-Verlag, New York, 1973.

20. A.J. Silberger, $P G L_{2}$ over the p-adics, Lecture Notes in Math., no. 166, Springer-Verlag, Berlin, 1970.

21. A.B. Venkov, Spectral theory of automorphic functions, Proc. Steklov Inst. Math. 1982, issue 4 (153).

22. J.-L. Waldspurger, Correspondance de Shimura, J. Math. Pures Appl. 59 (1980), 1-133.

23. __ Correspondance de Shimura et quaternions, Forum Math. 3 (1991), 219-307. 74078

Department of Mathematics, Oklahoma State University, Stillwater, Oklahoma

E-mail address: cogdellomath.okstate.edu

Department of Mathematics, Yale University, New Haven, Connecticut 06520

E-mail address: ilya@pascal.math.yale.edu 